\title{
UMA MOSTRA GERAL DE ASPECTOS INSERIDOS NA OBRA CHRONOGRAPHIA, REPORTORIO DOS TEMPOS... (1603)
}

\section{GENERAL SAMPLE OF ASPECTS INSIDE THE OEUVRE CHRONOGRAPHIA, REPORTORIO DE TEMPOS... (1603)}

\author{
Antonia Naiara de Sousa Batista \\ Instituto Federal de Educação, Ciências e Tecnologia do Ceará (IFCE) \\ Ana Carolina Costa Pereira ${ }^{2}$ \\ Universidade Estadual do Ceará (UECE)
}

\section{Resumo}

Na área da Educação Matemática é visível a produção de trabalhos envolvendo o uso de recursos da história para o ensino de aspectos matemáticos. Essa inserção de estratégias da história da matemática na sala de aula pode ocorrer por diferentes maneiras, dentre elas, podemos destacar o uso de fontes históricas para o ensino de conceitos matemáticos. Essas fontes históricas podem se destacar como fonte primária, secundária ou didática. Dentre esses tipos, este artigo traz uma fonte primária que tem como ponto central a apresentação e a descrição da obra Chronographia, Reportorio dos Tempos... (1603), de Manoel de Figueiredo. A mesma é a uma fonte histórica que abrange diversas áreas do conhecimento que estavam em desenvolvimento no século XVII, tais como, a cosmografia, a astrologia, a navegação, entre outras. Ademais essa obra traz três instrumentos, entre eles, a balhestilha, no qual é apresentada a sua fabricação e uso, o quadrante geométrico destinado para medições de alturas e um tratado sobre diversos tipos de relógios. Entretanto, queremos destacar que este trabalho é um recorte da dissertação que está sendo desenvolvida no Programa de Pós-graduação em Ensino de Ciências e Matemática (PGECM), do Instituto Federal de Educação, Ciência e Tecnologia do Ceará (IFCE), que visa investigar as potencialidades didáticas que emergem da fabricação da balhestilha, para a construção de uma interface que vise articular história e ensino de matemática, por meio de atividades didáticas. Para isso foi realizado uma pesquisa qualitativa de cunho documental e descritiva da obra estudada, direcionada para a estrutura, assuntos, teoremas e proposições e a linguagem contida na mesma. Desta forma, percebe-se que a obra abrange conhecimentos matemáticos que vão muito além do saber e fazer de sua época, perpassando por assuntos que estão interligados com outras áreas de conhecimentos e que podem ser trabalhados didaticamente nas aulas de matemática da Educação Básica. Assim, visamos com esse artigo ampliar os estudos com obras raras no intuito de conhecê-las mais profundamente, de maneira a explorar as potencialidades didáticas que emergem dos estudos desses instrumentos contidos nas mesmas para o ensino de matemática.

Palavras-chave: Ensino de Matemática; História da Matemática; Fonte histórica. Chronographia, Reportorio dos Tempos...; Balhestilha.

\footnotetext{
1 antonianaiarabatista@yahoo.com.br

2 carolina.pereira@uece.br
} 


\begin{abstract}
In the Mathematics Education field, it is noticeable the production of works involving the use of resources from the history for the teaching of mathematical aspects. This insertion of strategies of the history of mathematics in the classroom can occur in different ways, among them, we can highlight the use of historical sources for the teaching of mathematical concepts. These historical sources can stand out as a primary, secondary or didactic one. Among them, this paper has a primary source that has as its main point the presentation and description of the oeuvre Chronographia, Reportorio dos Tempos... (1603), by Manoel de Figueiredo. This is a source that covers several areas of knowledge that were developed throughout the seventeenth century, such as cosmography, astrology, navigation, among others. In addition, this book introduces three instruments, they are, the Cross-staff, in which is presented its manufacture and use; the geometric quadrant, used for measurements of heights; and a treatise on several sort of clocks. However, we want to emphasize that this paper is a part of a dissertation that has been carrying out in the Graduate Program in Science and Mathematics Teaching (PGECM) of the Federal Institute of Education, Science and Technology of Ceará (IFCE), which aims to investigate the didactic potentialities that emerge from the construction of the Cross-staff in order to construct an interface that aims to articulate history and mathematics through ludic activities. To do so, a qualitative research was carried out with a descriptive character of the work, addressed to its structure, contents, theorems, propositions and language. In this way, one can perceive that the oeuvre covers mathematical knowledge that goes beyond the knowing and doing of its time, going through subjects interconnected with other areas of knowledge that can be performed in Mathematics classes of Primary and Secondary Education. Therefore, we propose with this research paper to expand the studies with rare publications so as to know them deeply and explore the didactic potential that emerges from the studies of these instruments, which are inside of them, for the teaching of mathematics.
\end{abstract}

Keywords: Mathematics Teaching; History of Mathematics; Historical source. Chronographia, Reportorio dos Tempos ...; Cross-staff.

\title{
Introdução
}

$\mathrm{Na}$ área da Educação Matemática é notório que nos últimos decênios houve um aumento das discussões e reflexões relacionadas a assuntos, perspectivas e questões ligadas ao desenvolvimento do ensino e aprendizagem dos conceitos matemáticos, isso tanto no Brasil quanto no exterior (PAIS, 2002).

Baroni, Texeira e Nobre (2004), nos mostra que é nesse caminho de discussões que aflora o interesse dos professores pela história da matemática, culminando, portanto, em uma maior produção de artigos, contendo reflexões e ensaios acerca do uso da história da matemática na sala de aula e levantando diversos pontos a favor de sua aplicação no âmbito escolar.

Entretanto, sabemos que essa inclusão da história da matemática nas aulas de matemática não pode ocorrer de qualquer maneira, é preciso ter um conhecimento 
aprofundado, não só do conhecimento matemático que se pretende ensinar, mas é necessário se remeter ao "passado", por meio de leituras de obras primárias e secundárias, para que se possa compreender o processo de construção de um específico conhecimento matemático.

Desta forma, Baroni, Texeira e Nobre (2004), nos apresenta diversas maneiras de inserir a história da matemática na sala de aula, dentre elas: a produção de trabalhos orientados pela história da matemática; a utilização da história da matemática para aprimorar a construção de um conceito matemático; exploração de aspectos históricos da cultura matemática de um determinado povo; a utilização de fontes históricas originais; dentre outras.

Assim, neste trabalho iremos tratar sobre uma fonte histórica primária, chamada Chronographia, Reportorio dos Tempos..., de autoria de Manoel de Figueiredo, que na visão de Baroni, Texeira e Nobre (2004) para que a mesma seja utilizada no ensino de matemática é necessário um estudo árduo e intrínseco acerca da mesma. Além disso, os autores afirmam que, "em muitos casos, uma fonte requer um entendimento detalhado e profundo da época em que foi escrita, do contexto geral das ideias, além do entendimento da língua" (p. 174).

Para que isso ocorra é necessário realizar um levantamento de outras obras em torno do século em que a obra principal, em estudo, foi lançada, fazendo a análise não só de fontes que tratem de matemática, mas também que aborde outras áreas e aspectos relacionados a política, a economia, a parte cultural, entre outros.

Desde modo, este artigo apresenta e descreve a obra intitulada, Chronographia, Reportorio dos Tempos..., de autoria de Manoel de Figueiredo, que foi publicada no início do século XVII, mais especificamente, em 1603. Sua importância se dá pelas possíveis potencialidades didáticas que emergem da manipulação do instrumento, conhecido por balestilha, que se encontra inserida nessa fonte histórica primária.

\section{Marco Teórico}

São vários os autores que realizam pesquisas voltadas para uso de fontes históricas no ensino de matemática, dentre eles podemos destacar, Jahnke (2002), Pereira e Pereira (2015), Pinsky (2006), entre outros. Silva (2013, p. 38) ao trabalhar também com fontes históricas define a mesma como sendo, "vestígios do passado, 
deixados por sociedades a e agentes, que nos permitem desvendar seus hábitos, seus costumes, suas produções culturais e, principalmente, suas formas de organização".

Corroborando com essa definição Garnica e Souza (2012, p. 27) afirma que, "fontes seriam os resíduos do passado que chegam até nós" e exemplificam algumas delas, como sendo, fontes orais, arquitetônicas, escritas, iconográficas, entre outras, que podem ser mais especificamente, entrevistas, igrejas ou a fachada de escolas antigas, transcrição de uma gravação ou documento antigo, um registro fotográfico de um corpo docente de uma determinada escolar, respectivamente, dentre outros exemplos.

Entretanto, quando nos referimos à inserção da fonte histórica na educação matemática, a mesma pode ser classificada em três tipos: fonte primária, fonte secundária e fonte didática (TZANAKIS; ARCAVI, 2000). Os autores descrevem a fonte primária com sendo aquela extraída de obras matemáticas originais; enquanto que, a fonte secundária seria algum material que passou por um tratamento, como por exemplos, livros didáticos que trazem aspectos históricos, livros de história da matemática; e a fonte didática seria a retirada de aspectos da fonte primária e secundária que visassem construir uma abordagem mais dinâmica, "incluindo exposição, tutorial, exercício, etc" (p. 212).

Segundo Jahnke (2002), a fonte primária em contraposição a fonte secundária proporciona um conhecimento mais aprofundado e detalhado a respeito de um determinado tópico e, além disso, permite compreender a orientação histórica de um assunto, levando em conta certas interpretações, juízos de valores ou até mesmo falsas declarações expostas por outras concepções da história.

Assim, o trabalho com fontes históricas primárias possibilita se remeter ao período no qual as mesmas estavam inseridas e conhecer aspectos sociais, políticos, econômicos, religiosos, entre outros, que a influenciaram e tiveram grande importância, e que por consequência, ainda induzem mudanças no nosso período atual (PEREIRA; PEREIRA, 2015).

Dentre os vários tipos de fontes históricas já citadas, Pereira, Martins e Silva (2017) ampliam essa lista com "os papiros, tábuas, instrumentos de navegação e de medição, pinturas, artefatos históricos, entre outros". Desta maneira, a obra Chronographia, Reportorio dos Tempos... e os instrumentos inseridos na mesma, como a balestilha, se encaixam como fontes históricas que incorporam conhecimentos 
matemáticos e não matemáticos de uma determinada época, possibilitando assim, articular história e ensino.

\section{Metodologia}

Esse artigo se fundamenta em uma pesquisa qualitativa de cunho documental e descritivo da obra Chronographia, Reportorio Dos Tempos... (1603), de Manoel de Figueiredo, que segundo Gil (2002), consiste no trabalho com documentos que não receberam nenhum tratamento de análise ou critica, ou seja, arquivos primários, tais como, livros, documentos particulares, fotografias, vídeos, entre outros, que possibilitem fazer um reconhecimento mais detalhado das fontes escolhidas.

A mesma foi priorizada, por diversas vezes aparecer citada em pesquisas realizadas por autores brasileiros e internacionais, tais como Morey e Mendes (2005), Pinto (2010), Santos (2013). Entretanto, nenhum desses trabalhos utilizaram a obra original, ou seja, a fonte primária, como uma fonte histórica dentro do desenvolvimento de sua produção.

Segundo Figueiredo (1913), chronographia é um substantivo feminino que deriva-se da palavra chronologia, que significa "tratado das divisões do tempo" ou "tratado das datas históricas". Assim, para descrever essa obra, partimos do detalhamento da estrutura e dos assuntos que estão sendo abordados, dos teoremas e proposições e da linguagem contida na mesma.

\section{Apresentação e discussão dos dados}

A obra Chronographia, Reportorio dos Tempos..., impressa em 1602 e publicada só em 1603, na região de Lisboa, foi editada por Jorge Rodriguez e escrita por Manoel de Figueiredo, matemático e cosmógrafo-mor, nativo de Torres Novas. A mesma apresenta a seguinte capa (figura 1): 


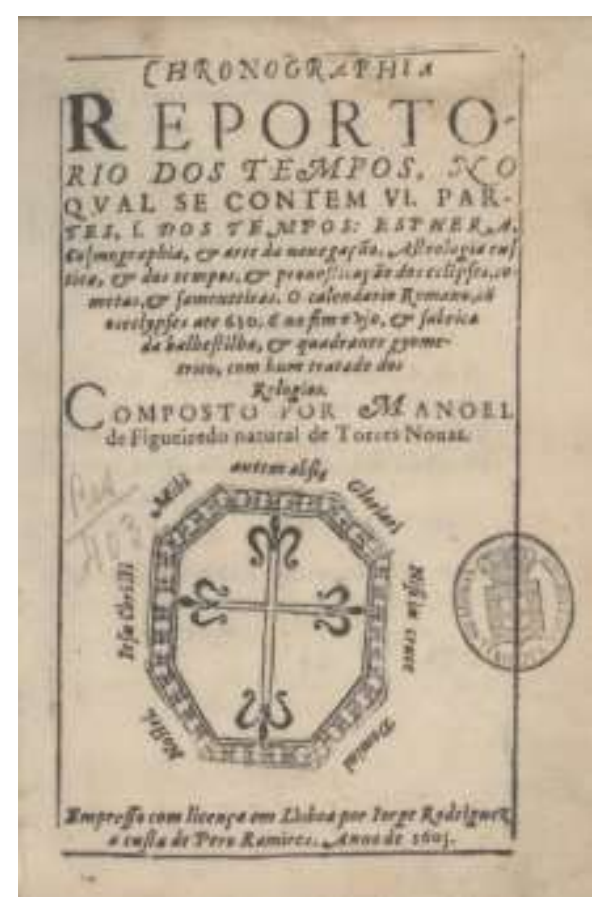

Figura 01 - Frontispício da Chronographia, Repertorio dos Tempos... (1603)

Fonte: Chronographia, Repertorio dos Tempos...(1603)

Neste livro faz-se a reunião e discussão de diversas áreas do conhecimento, tais como, a geografia, a cosmografia, a navegação, a astrologia, a topografia, entre outras, que podem ser constatadas a partir do título da obra, como podemos ver na figura 2.

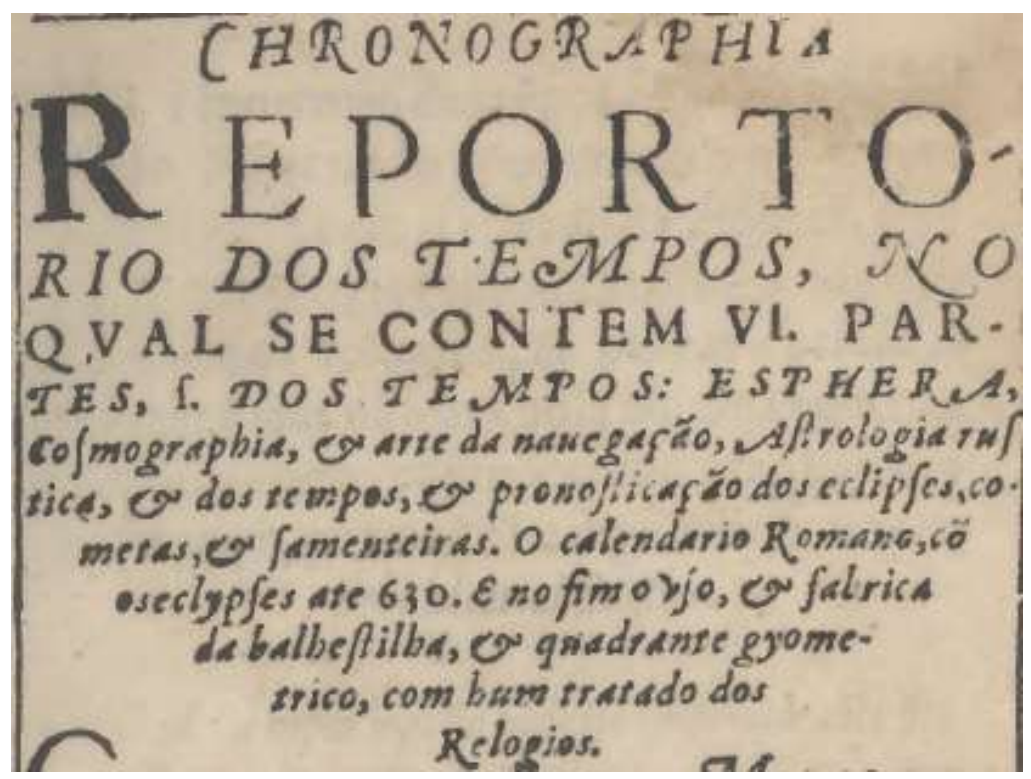

Figura 02 - Título e subtítulo da obra

Fonte: Chronographia, Repertorio dos Tempos...(1603) 
Por adaptação o título seria, "Chronographia Reportório dos tempos, no qual se contem 6 partes, dos tempos: esphera, cosmographia, e arte da navegação, astrologia rústica, e dos tempos, e pronosticação dos eclipses, cometas, e sementeiras. $O$ calendário Romano, com os eclipses ate 630. E no fim o uso, a fabrica da balhestilha, $e$ quadrante geométrico, com um tratado dos relógios". A obra possui um total 284 folhas ${ }^{3}$, que estão distribuídas em seis partes, conforme o expomos no quadro 1:

Quadro 1 - Descrição da obra

\begin{tabular}{|c|c|c|c|}
\hline PARTE & TÍTULO & $\begin{array}{c}\text { QTD. } \\
\text { CAPÍTULOS }\end{array}$ & $\begin{array}{c}\text { QTD. } \\
\text { FOLHAS }\end{array}$ \\
\hline Primeira & Do tempo e suas partes & 38 & 40 \\
\hline Segunda & $\begin{array}{l}\text { Da astronomia, na qual se trata do } \\
\text { céu, e de suas partes, e de como nele pois } \\
\text { DEUS o tempo, juntamente com todos os } \\
\text { seus movimentos, estrelas, planetas, orbes, } \\
\text { eixos, polos, círculos da esfera, e com todas } \\
\text { as mais coisas que DEUS nele criou, } \\
\text { ordenou }\end{array}$ & 32 & 77 \\
\hline Terceira & $\begin{array}{l}\text { Da geografia em que declaramos a } \\
\text { terra, a qual teve o terceiro lugar nas } \\
\text { palavras da sagrada escritura, DEUS criou } \\
\text { o céu, e a terra }\end{array}$ & 22 & 33 \\
\hline Quarta & $\begin{array}{l}\text { Da astrologia rústica, muito } \\
\text { necessária para a agricultura, e para todo o } \\
\text { lavrador curioso amigo da lavoura, e com } \\
\text { um tratado muito necessário, e proveitoso a } \\
\text { saúde humana para os físicos, surgiães, e } \\
\text { sangradores, e pronosticação dos eclipses } \\
\text { do sol, e da lua }\end{array}$ & 47 & 69 \\
\hline Quinta & $\begin{array}{c}\text { Do calendário, epacta, número } \\
\text { áureo, endiçam, temporas, e da } \\
\text { pronosticação dos } 12 \text { meses do ano, e do } \\
\text { lunário de } 603 \text { até } 630 \text { com os eclipses no } \\
\text { cabo do lunário, e suas significações. }\end{array}$ & 34 & 48 \\
\hline Sexta & $\begin{array}{l}\text { Da fabrica, e uso da balhestilha, ou } \\
\text { radio astronômico, e do uso e fabrica, do } \\
\text { quadrante geométrico e da fabrica, e uso } \\
\text { dos relógios horizontais, verticais, laterais, } \\
\text { equinociais, polares declinantes a todas as } \\
\text { partes do mundo, e inclinantes }\end{array}$ & 12 & 19 \\
\hline
\end{tabular}

Fonte: Figueiredo (1603)

\footnotetext{
3 A obra Chronographia, Repertorio dos Tempos...(1603) apenas apresenta numeração relacionada a
} folha que está sendo usada. 
De acordo com o quadro 1 é possível perceber que a obra traz vários tópicos que culminam em campos específicos do conhecimento, como por exemplo, questões sobre cosmografia, trazendo assuntos sobre o céu, planetas, signos, esfera natural, entre outros. Ou a geografia da terra, abrangendo itens sobre latitude, longitude, climas, etc. Juntamente, com a astrologia e sua influência no corpo humano, na agricultura e nos eventos naturais.

Além disso, faz menção há três instrumentos, entre eles a fabricação e uso da balestilha ou radio astronômico destinada para aplicações na astronomia; a fabricação e uso do quadrante geométrico designado para medir alturas de qualquer grandeza; e por fim, um tratado que apresenta a fabricação de diferentes relógios, desde horizontais até verticais.

Ademais, essa obra apresenta um vasto conhecimento de definições e proposições relacionadas à geometria euclidiana plana, juntamente com algumas construções geométricas, que para a época davam suporte a fabricação e uso desses instrumentos, inclusive da balestilha. A linguagem da mesma encontra-se inserida na transição do português arcaico para o português moderno. É possível perceber a presença de alguns vocábulos em latim, tais como: creavit, celum e terram.

\section{Considerações finais}

A fonte histórica é um recurso que possibilita nas aulas de matemática explorar diversos conteúdos matemáticos que estão agregados ao contexto no qual a obra estava inserida, podendo assim, articular não só conhecimentos matemáticos, mas também aspectos contextuais, tais como, sociais, políticos, religiosos, econômicos, entre outros, que permearam o estudo desses conceitos e da própria obra.

Diante do estudo que está sendo realizado percebemos que, a Chronographia, Reportotio dos Tempos..., é uma obra que trouxe para sua época diversos saberes acerca de áreas do conhecimento que se encontravam em construção no período de sua publicação. Além de, apresentar a fabricação e uso de diferentes instrumentos que estavam voltados para alguns desses campos, em destaque, a balestilha ou radio astronômico, um instrumento voltado para uso na náutica e também na astronomia e, que foi bastante utilizado, no período pré e pós publicação da obra.

Deste modo, consideramos que essa obra é potencialmente didática para ser utilizada na sala de aula, para o estudo de conhecimentos e construções geométricas, 
principalmente, quando tratamos da fabricação e uso da balestilha. Entretanto, precisase de um debruçamento maior, com foco nesse instrumento, para o estudo aprofundado de suas potencialidades que podem ser atreladas ao âmbito escolar, no intuito de articular história e ensino de matemática.

Assim, esse artigo apresenta um recorte da minha pesquisa de mestrado, desenvolvida no Programa de Pós-Graduação em Ensino de Ciências e Matemática, do Instituto Federal de Educação, Ciência e Tecnologia do Ceará, que tem como foco investigar as potencialidades didáticas que emergem da fabricação da balestilha, para a construção de uma interface que articule história e ensino de matemática, embasada nos estudos de Saito e Dias (2013). Visando desta forma, contribuir para dar continuidade às pesquisas que fazem uso de fontes históricas primárias, em especial, com instrumentos, para articular história e ensino de matemática.

\section{Referências}

BARONI, Rosa Lúcia; TEXEIRA, Marcos Vieira; NOBRE, Sergio Roberto. A investigação científica em história da matemática e suas relações com o programa de pós-graduação em educação matemática. In: BICUDO, Maria Aparecida Viggiani; BORBA, Marcelo de Carvalho. Educação matemática: pesquisa em movimento. São Paulo: Cortez Editora, 2004. p. 164-185.

FIGUEIREDO, Manoel de. Chronographia Reportorio dos tempos, no qual se contem VI. partes, f. dos tempos: esphera, cosmographia, e arte da navegação, astrologia rustica, e dos tempos, e pronosticação dos eclipses, cometas, e sementeiras. $\mathrm{O}$ calendario Romano, com os eclypses ate 630 . E no fim o uso, a fabrica da balhestilha, e quadrante gyometrico, com hum tratado dos relogios. Lisboa. 1603.

FIGUEIREDO, Candido de. Novo Diccionário da Língua Portuguesa. 2. ed., 1913. Disponível em: <http://dicionario-aberto.net/dict.pdf>. Acesso em: 25 set. 2017.

GARNICA, Antonio Vicente Marafioti; SOUZA, Luzia Aparecida de. Elementos da história da Educação Matemática. São Paulo: Cultura Acadêmica, 2012.

GIL, Antonio Carlos. Como elaborar projetos de pesquisa. 4. ed. São Paulo: Editora Atlas, 2002. $175 \mathrm{p}$.

JAHNKE, Hans Niels. The use of original sources in the mathematics classroom. In: FAUVEL, John; VAN MAANEN, Jan. History in Mathematics Education. New York, Boston, Dordrecht, London, Moscow: Kluwer Academic Publishers, 2002. Cap. 9. p. 291-328.

MOREY, Bernadete; MENDES, Iran Abreu. Conhecimentos matemáticos na época das navegações. Rio Grande do Norte: Sbhmat, 2005. 54 p. 
PAIS, Luiz Carlos. Introdução. In: MACHADO, Silvia Dias Alcântara et al. Educação Matemática: Uma introdução. 2. ed. São Paulo: Educ, 2002. Cap. 2. p. 9-12.

PEREIRA, Ana Carolina Costa; PEREIRA, Daniele Esteves. Ensaio sobre o uso de fontes históricas no ensino de Matemática. REMATEC. Revista de Matemática, Ensino e Cultura (UFRN), v. 10, p. 65-78, 2015.

PEREIRA, Ana Carolina Costa; MARTINS, Eugeniano Brito; SILVA, Isabelle Coelho da. Evolução histórica da multiplicação do século X ao XVI: Construindo interfaces para o ensino. Belém: Sociedade Brasileira de Educação Matemática, 2017. 70 p.

PINSKY, Carla Bassanezi (Org.). Fontes Históricas. São Paulo: Contexto, 2006, p. 203-233.

PINTO, Margarida Matias. Os instrumentos náuticos de navegação e o ensino da geometria. Lisboa: Sociedade Portuguesa de Matemática, 2010. 80 p.

SANTOS, Franjossan Gomes dos. Cálculo de distâncias com a Balestilha. 2013. 58 f. Dissertação (Mestrado) - Curso de Mestrado Prossional em Matemática, Centro de Ciências da Natureza, Universidade Federal do Piauí, Teresina, 2013.

SAITO, Fumikazu; DIAS, Marisa da Silva. Interface entre história da matemática e ensino: Uma atividade desenvolvida com base num documento do século XVI. Ciência e Educação, São Paulo, v. 19, n. 1, p.89-111, jan. 2013.

SILVA, Ana Paula Pereira do Nascimento. A leitura de fontes antigas e a formação de um corpo interdisciplinar de conhecimentos: Um exemplo a partir do Almagesto de Ptolomeu. 2013. 100 f. Dissertação (Mestrado em Ensino de Ciências Naturais e Matemática) - Universidade Federal do Rio Grande do Norte, Natal, 2013.

TZANAKIS, Constantinos; ARCAVI, Abraham. Integrating history of mathematics in the classroom: an analytic survey. In: FAUVEL, John; MAANEN, Jan van. History in Mathematics Education: The ICMI Study. New York/Boston/

Dordrecht/London/Moscow: Kluwer Academic Publishers, v. 6, 2000. p. 201- 240. 